\title{
$\mathrm{CiSj}$
}

\section{AN EFFICIENT AND SECURED NON BLIND WATERMARKING SCHEME FOR COLOR IMAGES USING DWT AND ARNOLD TRANSFORM}

\author{
Nagaraj V. Dharwadkar, B.B. Amberker \\ Computer Science and Engineering, National Institute of Technology, Warangal, (AP), India \\ e-mail: nvd@nitw.ac.in,bba@nitw.ac.in
}

\begin{abstract}
In the early days, encryption and access control techniques are used to protect the ownership of image. Recently, the watermarking schemes are utilized to define the copyright of image. Digital watermarking is a concept of embedding a special security pattern into cover image. It has recently become important in various application areas of image processing. In this paper we present a new invisible, robust, non blind frequency domain watermarking scheme. This scheme embeds the monochrome (logo) watermark into the high and middle $\{H L, L H, H H\}$ frequency bands of Arnold transformed luminance channel of the color image. The security of scheme is improved by scrambling the luminance channel of the color image using Arnold transform. In order to increase the detection speed and efficiency of algorithm, the location of modified high and middle frequency components are stored into key array. Use of this key array improves the speed of the extraction algorithm. The experimental results show that the watermark is robust against different types of attacks like image cropping, image filtering, image Compression, image blurring, and Image transformations. Further, the results of the proposed scheme are analyzed on the set of images.
\end{abstract}

Keywords: Watermarking, DWT, DRM, Copy Right Protection, RGB, YIQ.

\section{INTRODUCTION}

Due to rapid growth of multimedia applications over Internet, we have seen an explosion of data in the Internet and the extensive use of digital media. Consequently, digital data owners can transfer multimedia documents across the Internet easily. Therefore, there is an increase in the concern over copyright protection of digital content $[1,2]$. In the early days, encryption and control access techniques were employed to protect the ownership of media. However, to protect against unauthorized copying after the media have been successfully transmitted and decrypted, recently the watermarking techniques are utilized [3], because watermarking algorithms embed the watermark into digital data and using these watermark we can prevent the unauthorized copying.

A digital watermark is a pattern of bits inserted into an image, audio or video file. The name comes from the barely visible text or graphics imprinted on stationery that identifies the manufacturer of the stationery. There are several applications of watermarking such as, Broadcast monitoring, Owner identification, Proof of ownership, Transaction tracking, Content authentication, Copy control, and Device control $[4,5]$.

The watermarking techniques proposed in the literature fall in two categories: spatial-domain methods [4] and transform-domain methods [7, 8, $12,14,15$ and 16]. Many techniques have been proposed in the spatial domain, such as the LSB (least significant bit) insertion method [9], the patchwork method [4] and the texture block coding method [4]. These techniques process the location and luminance of the image pixel directly. The LSB method has a major disadvantage that the least significant bits may be easily destroyed such as randomly flipping the lower bits or lossy compression.

A transform-domain method, such as the Fourier Transform [10], Discrete Cosine Transform, [11, 13], or Discrete Wavelet Transform [12, 14, 15, 16], are based on special transformations, and processes the coefficients in the frequency domain for hiding data. In these methods the watermark is hidden in the high frequency coefficients [12] or middle frequency coefficients [13] of the protected image. The low frequency coefficients are more likely to be suppressed by filtration as noise. Therefore, the high frequency coefficients of the protected image are used to embed the watermark [12]. How to select the best frequency portions of the image for hiding watermark is an important and difficult topic. The transform-domain method is more robust than the spatial-domain method against compression, 
cropping, and jittering [14]. The robustness is maintained at the price of imperceptibility in the transform domain.

In this paper, we propose a watermarking algorithm in which the color image is transformed into Red (R), Green (G) and Blue (B) channels. Further, these channels are transformed into Luminance (Y), Intensity (I) and Hue (Q) channels. The Arnold transformation is applied $\mathrm{T}$ times on Luminance $(\mathrm{Y})$ channel of image, which produces the scrambled Luminance (Y'). The Discrete Wavelet Transformation (DWT) is applied on Y' channel. The high and middle frequency components of this $Y^{\prime}$ channel are modified to embed the watermark. The locations of modified frequency bands are stored into an array called key array. The inverse DWT is applied on this modified Y' channel to get watermarked $\mathrm{Y}^{\prime}$ channel. On this watermarked $Y^{\prime}$ channel T times the inverse Arnold transformation is applied to get watermarked Luminance $Y^{\prime}$. The watermarked Luminance Y', original I and Q are transformed into Color image. The high and middle frequency bands are used in embedding, because these bands contain the edge information of image, and human vision system is less sensitive to change in edge. In the extraction algorithm the watermarked image is transformed into RGB channels. The RGB channels are transformed into YIQ, and then the $T$ times Arnold transform is applied on Y channel. The DWT is applied on Arnold transferred Y channel. To extract the watermark the modified high and medium frequency components are selected using the key array. The correlation between extracted watermark and original watermark is calculated for objective judgment of the extraction fidelity. The use of key array in extraction algorithm helps to easily identify the frequency components used for embedding the watermark, thus increases the speed of extraction. The use of Arnold transfer increases the security of image.

The paper is organized as follows: In section 2, we explain the Arnold transform. In Section 3, we describe the Discrete Wavelet Transformation. In Section 4, we describe the proposed watermark embedding extraction model. In Section 5, we present our results. Finally, in Section 6, we conclude our paper. Related references are listed in Section 7.

\section{ARNOLD TRANSFORM}

In order to enhance the security and robustness, we process the original image $I$ with Arnold transform to obtain the scrambled image [17]. The transform is a process of clipping and splicing that re-align the pixel matrix of digital image. The
Arnold transform is shown as follow

$$
\left[\begin{array}{l}
x^{\prime} \\
y^{\prime}
\end{array}\right]=\left[\begin{array}{ll}
1 & 1 \\
1 & 2
\end{array}\right]\left[\begin{array}{l}
x \\
y
\end{array}\right] \bmod (N)
$$

Where $x$ and $y$ are the pixel position of original image, $x^{\prime}$ and $y^{\prime}$ are the pixel position of scrambled image. $N$ is the height or width of the image processed. The Arnold transform changes the pixel position for several times, a scrambled image can be generated as follows

$$
Q_{\left(x^{\prime}, y^{\prime}\right)}^{T+1}=A Q_{(x, y)}^{T} \bmod (N)
$$

$Q_{\left(x^{\prime}, y^{\prime}\right)}^{T+1}$ and $Q_{(x, y)}^{T}$ are the pixels of original image and transformed images respectively, $A$ is a Arnold transform matrix and $T$ is the number of times of the Arnold transform.

\section{DISCRETE WAVELET TRANSFORMATION}

The Discrete Wavelet Transform of an image divides the image into bands of approximately equal bandwidth on a logarithmic scale. This is similar to the retina of the human eye that splits the image into several components of each having a bandwidth of approximately equal to one octave. It is believed that the use of DWT for watermarking produces an imperceptible watermark [14].

The basic idea of the DWT for a two-dimensional image is described as follows. An image is first decomposed into four parts of high, middle, and low frequency subcomponents (i.e., $L L_{1}, H L_{1}, L H_{1}, H H_{1}$ ) by critically subsampling horizontal and vertical channels using subcomponent filters. The subcomponents labeled $H L_{1}, L H_{1}$, and $H H_{1}$ represent the finest scale wavelet coefficients. To obtain the next coarser scaled wavelet components, the subcomponent $L L_{1}$ is further decomposed and critically subsampled. This process is repeated several times, which is determined by the application at hand. An example of an image being decomposed into ten subcomponents for three levels is shown in Figure 1.

We used third level DWT decomposition which increases the Signal to Noise Ratio by reducing the effect of noise on cover image [16]. To embed the watermark high frequency components are considered, since high frequency components contains edge information and the human eye is less sensitive to changes in edges. In watermarking algorithms, the main concern besides invisibility of the watermark is how to choose the frequency components to embed the watermark such that it will survive the possible attacks that the transmitted image may undergo. 


\begin{tabular}{|c|c|c|c|}
\hline & HL3 & \multirow{2}{*}{ HL2 } & \\
\cline { 1 - 1 } LH3 & HH3 & \\
\cline { 1 - 1 } LH2 & HH2 1 \\
& & \\
& & \\
I.H1 & HH1 \\
\hline
\end{tabular}

Fig. 1 - Third Level DWT Decomposition

\section{PROPOSED MODEL}

The proposed DWT- scheme is a non-blind image watermarking scheme, where in it embeds the monochrome image (Logo) into a selected set of DWT coefficients. The embedding and extraction algorithms are shown in Figure $2 \mathrm{a}$ and Figure 2b, respectively.

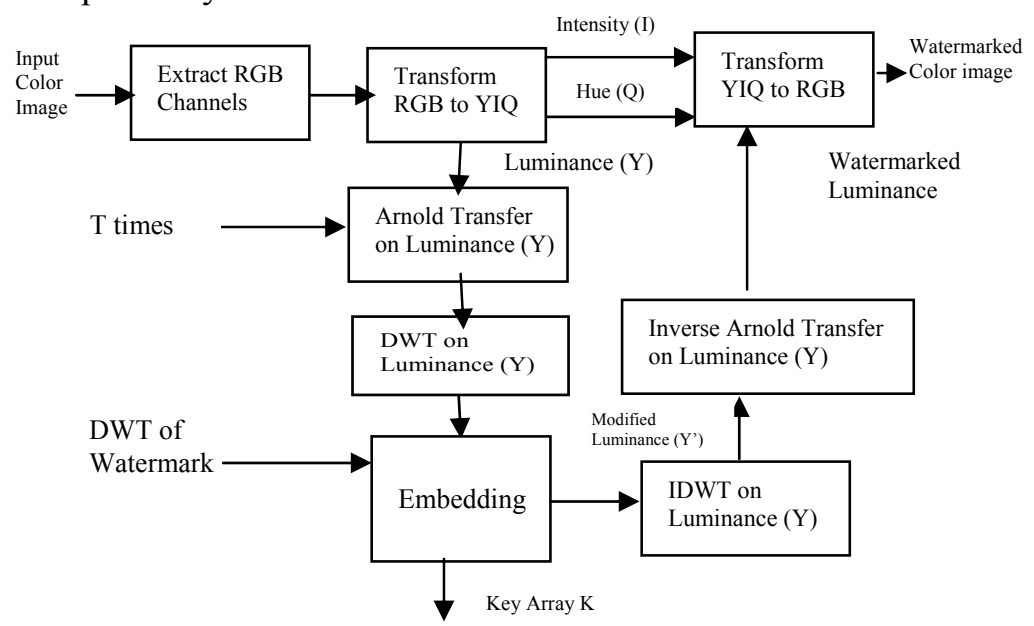

Fig. 2a - Watermark Embedding Algorithm

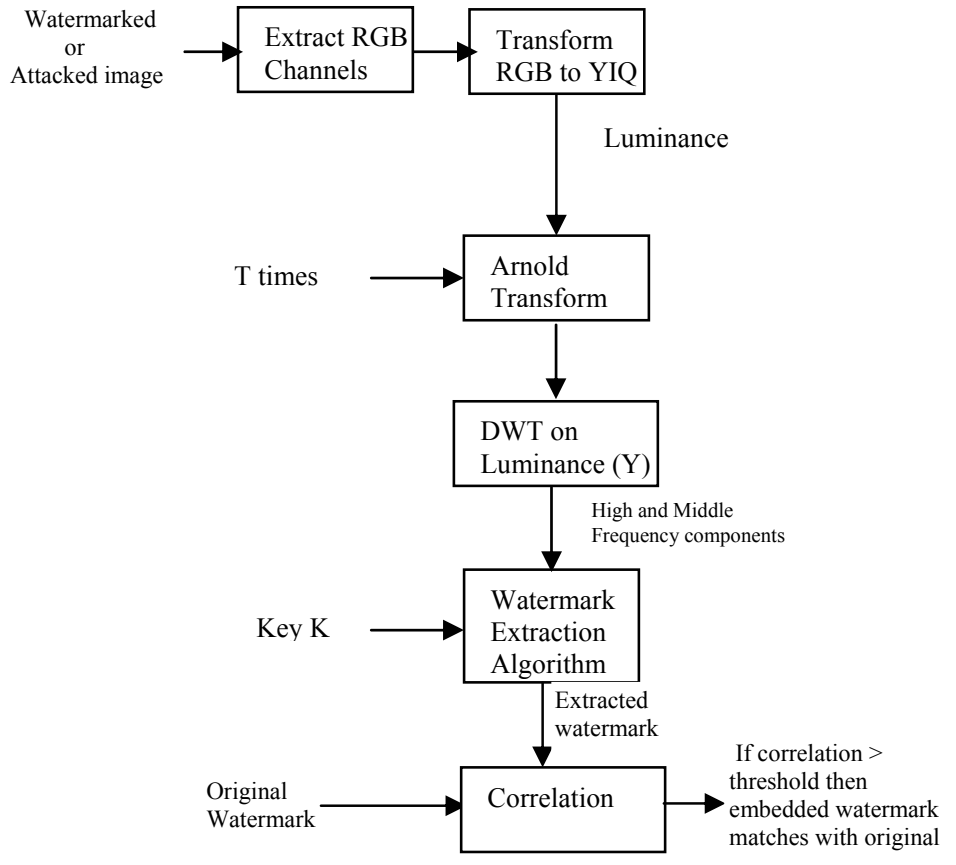

Fig. 2b - Watermark Extraction Algorithm

\section{A. WATERMARK EMBEDDING ALGORITHM}

As shown in Figure 2a the R, G, B channels are extracted from color image, and then these R, G, and B channels are transformed to Y, I, Q channels. The Arnold transform is applied $T$ time on the $\mathrm{Y}$ channel. To embed the watermark, only high frequency components of Arnold transformed luminance channel of image are considered because high frequency luminance components are less sensitive to human eye. The DWT is applied on scrambled Luminance channel of cover image, and watermark is embedded in high and middle frequency components. The location of the modified high frequency components are maintained in key array $K$. The inverse DWT is applied on watermarked Luminance channel, and then $T$ time inverse Arnold transform is applied on this luminance channel. Later this channel is combined with Intensity and hue channels to get the watermarked color image. The watermark embedding algorithm can be summarized as follows:

Algorithm: Watermark Embedding Input: cover image (color), watermark (monochrome) image and $T$ number of Arnold transform.

Output: Watermarked color image.

1. Read color (cover) image $I$ of size $N \times N$.

2. Read the watermark (monochrome) image $X$ of size $M \times M$ where $X(\mathrm{i}, \mathrm{j}) \in$ $\{0,1\}, i=1,2, \ldots, M, j=1,2$, ..., $M$. Apply DWT on $X$ to get $D=\left\{d_{i j}\right\}$ of size $M \times M$.

3. Compute $R, G, B$ channels of size $N \times N$ from color image $I$.

4. Transform these $R, G, B$ channels into $Y, I, Q$ channels.

5. Apply $T$ times the Arnold transform on $Y$ channel.

6. The frequency subcomponents $\left\{H_{1}, H L_{1}, L H_{1}, \quad\left\{\left\{H_{2}, H L_{2}\right.\right.\right.$, $\left.\left.\left.L_{2},\left\{H_{3}, H L_{3}, L H_{3}, L L_{3}\right\}\right\}\right\}\right\}$ are obtained by computing the third level DWT of the $Y$ channel

7. Embed the watermark components into the frequency subcomponents, starting from $H H_{1}$ for each row select the frequency coefficients in descending order with respect to their absolute values. Modify 
each frequency coefficient $f$ to $f^{\prime}=f+\alpha d_{i j}$, Where $\propto$ is Watermark scaling factor, and $d_{i j}$ is watermark frequency coefficient. If the $H_{1}$ subcomponent is not sufficient to embed the complete watermark, then go for next subcomponents in a sequence $\left\{L H_{1}\right.$, $H_{1}, \quad\left\{\left\{H_{2}, \quad H L_{2}, \quad L H_{2}, \quad\left\{H_{3}, \quad L H_{3}\right.\right.\right.$, $\left.\left.\left.\left.\mathrm{HH}_{3}\right\}\right\}\right\}\right\}$.

8. Save the location of the modified frequency components into a key array $K$ of size $N \times$ $N$. The key array $K$ has value one if the coefficient is modified and zero if not.

9. Replace $\{f\}$ by $\left\{f^{\prime}\right\}$ in decomposed $\mathrm{Y}$ channel.

10. Compute Inverse DWT of modified $\mathrm{Y}$ channel.

11. Apply $T$ time inverse Arnold transform on modified $Y$

12. Combine modified $Y$ channel with I and Q to get watermarked color image

\section{Input:}

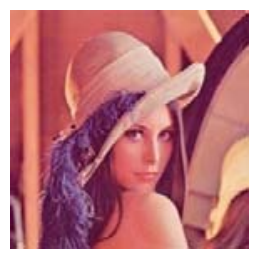

\section{NITW}

Fig. 3a - Original Image

Fig. $3 b$ - Watermark image

\section{Output:}
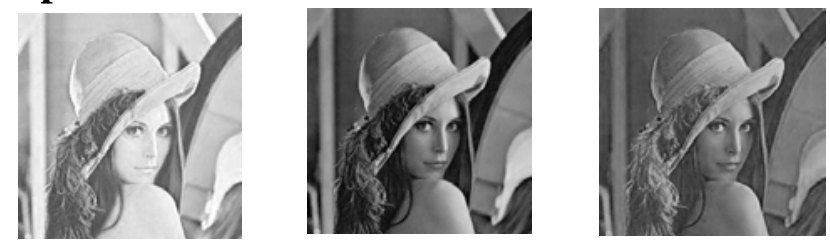

Fig. 3c - Red, Green and Blue Components of color image
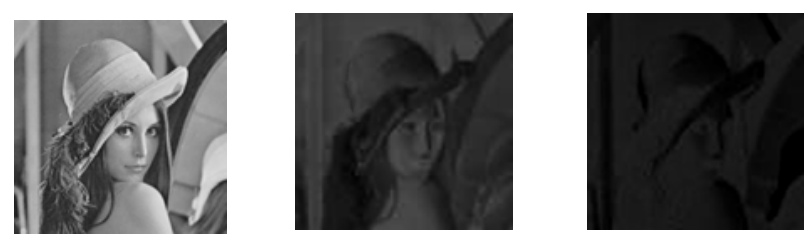

Fis. 3d - Luminance, Intensity and Hue Components of color Image

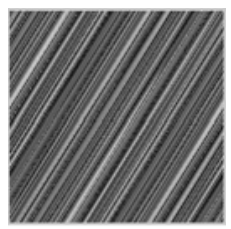

Fig. 3e - Arnold transformed Luminance for 4 times

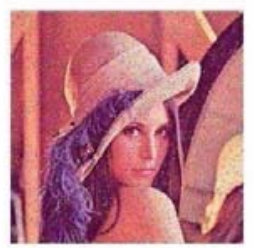

Fig. 3e - Watermarked Color image

\section{B. WATERMARK EXTRACTION ALGORITHM}

As shown in Figure $2 b$ the R, G, B channels are extracted from the watermarked color image. These channels are transformed into Y, I, Q channels. To extract the watermark, only luminance channel of image is considered. $T$ times the inverse Arnold transform is applied on Y channel. The DWT is applied on this scrambled Luminance channel, and from this, only the modified high and middle frequency components are retrieved using the key array $K$. These frequency components are used to extract the watermark. The correlation between extracted watermark and available watermark is calculated. If the extracted watermark and available watermark are tightly correlated then it indicates the embedded watermark matches with original watermark.

The watermark extraction algorithm is summarized as follows:

Algorithm: Watermark Extraction

Input: Watermarked image, cover (color) image and $T$ number of Arnold transform.

Output: Watermark,

1. Read the Watermarked image I' of size $N \times N$.

2. Compute R', G', B' channels of size $N \times N$ from watermarked color image $I^{\prime}$.

3. Transform these $R^{\prime}, G^{\prime}, B^{\prime}$ channels into $Y^{\prime}, I^{\prime}$, Q' channels.

4. The Arnold transform is applied $T$ times on the $Y^{\prime}$ channel

5. The frequency subcomponents $\left\{H H_{1}, H L_{1}, L H_{1}\right.$, $\left.\left\{\left\{H_{2}, H L_{2}, L H_{2},\left\{H_{3}, H L_{3}, L H_{3}, L L_{3}\right\}\right\}\right\}\right\}$ are obtained by computing the third level DWT of the $Y^{\prime}$ channel

6. The frequency subcomponents $\left\{H H_{1}, H L_{1}, L H_{1}\right.$, $\left.\left\{\left\{H_{2}, H L_{2}, L H_{2},\left\{H H_{3}, H L_{3}, L H_{3}, L L_{3}\right\}\right\}\right\}\right\}$ are obtained by computing the third level DWT of the Arnold transformed $Y$ channel of the unwatermarked color image.

7. Extract the watermark bits from the frequency subcomponents, starting from $\mathrm{HH}_{1}$ and using key array $K$ as $X_{\mathrm{ij}}{ }^{\prime}=\left(f^{6}-f\right) / \propto$. If $X_{\mathrm{ij}}{ }^{\prime}>S$, then $X_{\mathrm{ij}}{ }^{\prime}=1$ else $X_{\mathrm{ij}}{ }^{\prime}=0$, Where $i$ $=1,2, \ldots, M$, and $j=1,2, \ldots, M, f=$ frequency coefficient of $Y$ at the corresponding level and subcomponent, $f{ }^{\prime}=$ frequency 
coefficient of $Y^{\prime}$ at the corresponding level and subcomponent, $S$ is between 0 and 1 and $\alpha$ is scaling factor.

\section{Input:}

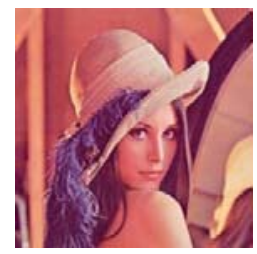

Fig. 4a - Original Image

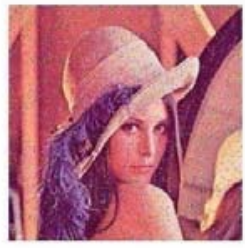

Fig. 4b - Watermarked Image

\section{Output:}
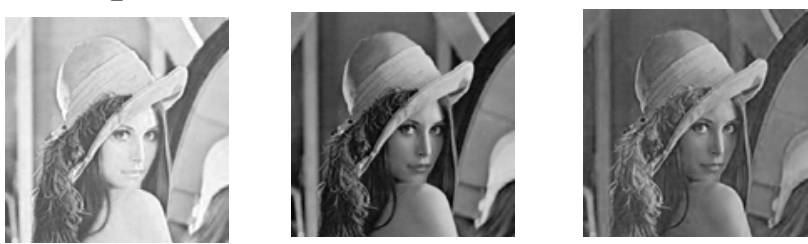

Fig. 4c - Red, Green and Blue Components of Watermarked image
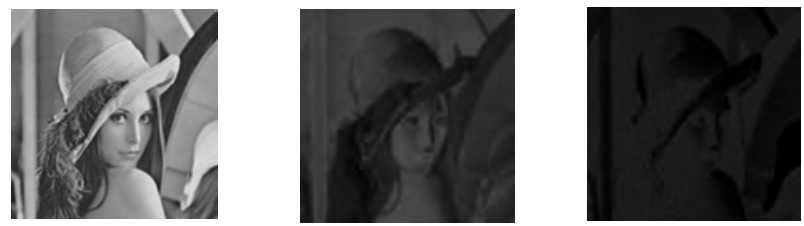

Fig. 4d - Luminance, Intensity and Hue Components of watermarked image

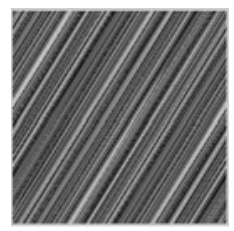

Fig. 4e - Arnold transformed Luminance for 4 times

$$
\text { NITIS }
$$

Fig. 4f - Extracted Watermark

\section{RESULTS AND DISCUSSIONS}

To analyse the algorithm, the MATLAB modules are defined separately for embedding and extraction of watermark. A $256 \times 256$ sized color image of Lena, Pepper, Baboon and House are taken as a cover image and the monochrome logo pattern of size $60 \times 70$, as a watermark. Figure 5 shows the figures considered in our experiments. Figure 6 shows the watermarked images. Various experiments are conducted on watermarked image to test the robustness of algorithm. Also, image processing operations like cropping, filtering, compression, adding noise, blurring and geometric transformations are applied on watermarked image. Then the qualities of extracted watermark are compared with original watermark using Normalized Correlation. The performance of embedding algorithm is measured using Signal to Noise Ratio metric.

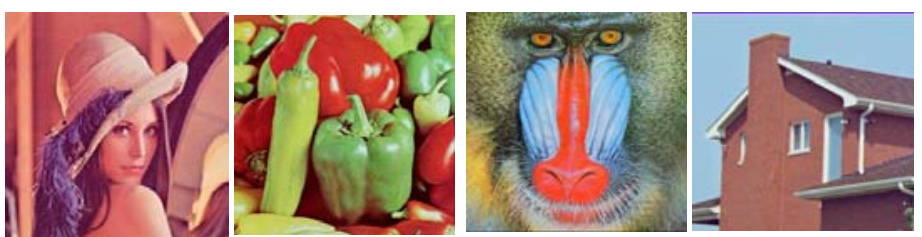

Fig. 5 - Cover images: Lena, Pepper, Baboon, House
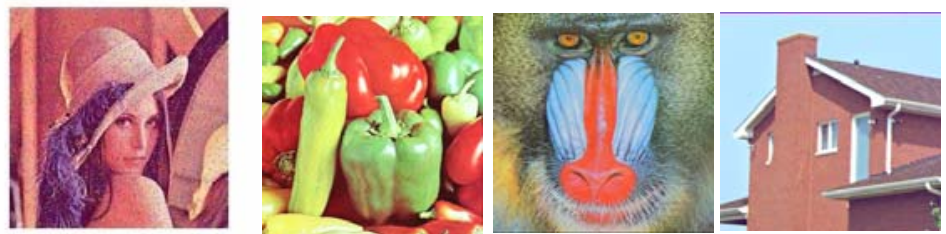

Fig. 6 - Watermarked images: Lena, Pepper, Baboon, House

\section{A. ROBUSTNESS AGAINST IMAGE CROPPING}

Image cropping is applied on watermarked image by cropping $20 \%$ of image. This is shown in Figure 7a.Then the extraction algorithm is applied to this cropped image. The extracted watermark is shown in the Figure $7 \mathrm{~b}$. The robustness of the algorithm is tested by applying different percentage of cropping. It is found that the quality of extracted watermark decays as the percentage of cropping increases. We get good quality of extracted watermark if the cropping is less than $40 \%$ as shown in Figure $7 \mathrm{c}$.

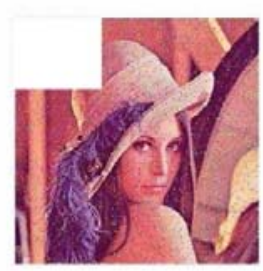

Fig. 7a-Cropped image

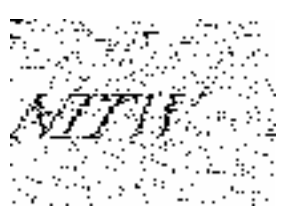

Figure 7b: Extracted Watermark after cropping 


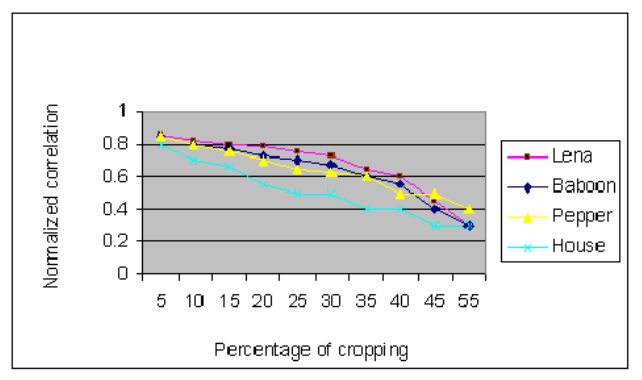

Fig. 7c - Effect of cropping

\section{B. ROBUSTNESS AGAINST COMPRESSION}

We use JPEG compression to check the robustness of algorithm against lossy compression. As shown in Figure 8, the quality of compressed image purely depends on the value of compression ratio. If the value of compression ratio is less than 6 , then the normalized correlation between extracted and original watermark is nearly 0.8 , thus image quality is also good. As the compression ratio increases the quality of the extracted watermark decays. The correlation coefficient is closer to 1 .

\section{ROBUSTNESS AGAINST FILTERING}

Image filtering is one of image processing method that is applied to remove the noise. Gaussian filters are important in many signal processing, image processing, and communication applications. These filters are characterized by narrow bandwidths, sharp cutoffs, and low overshoots. A key feature of Gaussian filters is that it has the same response shape in both time and frequency domains. The Gaussian filter is applied on the watermarked image. The filtered image is as shown in Figure 9a. The watermark is extracted from this filtered image is shown in Figure 9b. The Normalized correlation between extracted watermark and original watermark is 0.878 . This represent the algorithm is robust against filtering

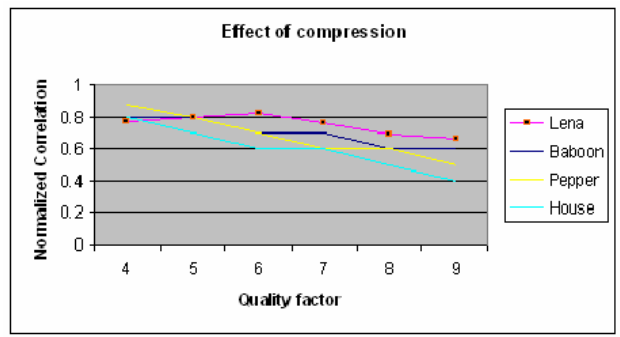

Figure 8: Effect of Compression
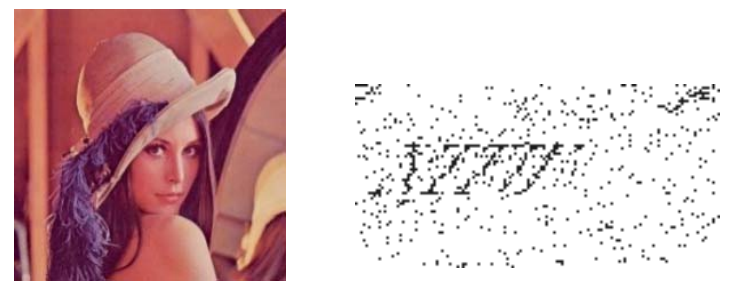

Fig. 9a - Low-Pass filtered Watermarked image

Fig. 9b- Extracted Watermark

\section{ROBUSTNESS AGAINST GEOMETRICAL TRANSFORMATION}

The geometric transformations are important manipulations of image. The transformations that modify the size of image is known as scaling, and the transformations that modify the angle of orientation is known as rotation. Geometrical transformation affects the quality of extracted watermark. The effect of different transformation on the watermarked image by measuring the Normalized correlation between the original watermark and extracted watermark is shown in Figure 10 to Figure 11. For the effect of scaling, we found the correlation is consistent for the scale factor of range 0.10 to 0.30 , for the effect of rotation, we found the correlation is good for the rotation angle must in the range 1 to 14 degrees.

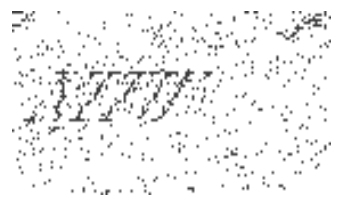

Fig. 10 - Extracted watermark from 0,2 scaled watermarked image and correlation is nearly 0.7705

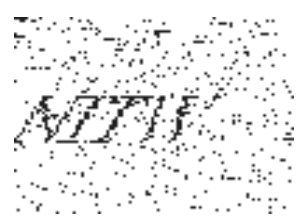

Fig. 11 - Extracted watermark from 6 degree Rotation of Watermarked image and correlation is nearly 0.7854

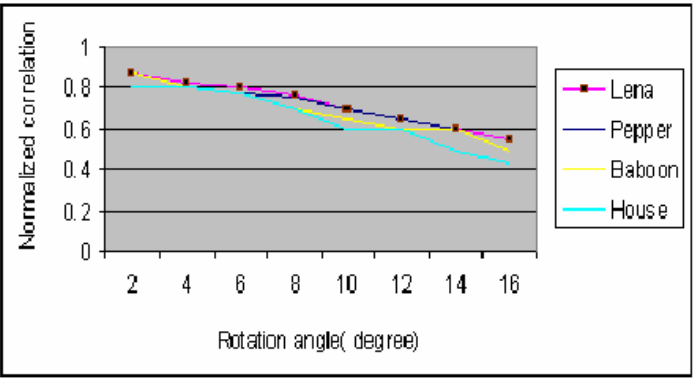

Fig. 12 - Effect of Rotation 


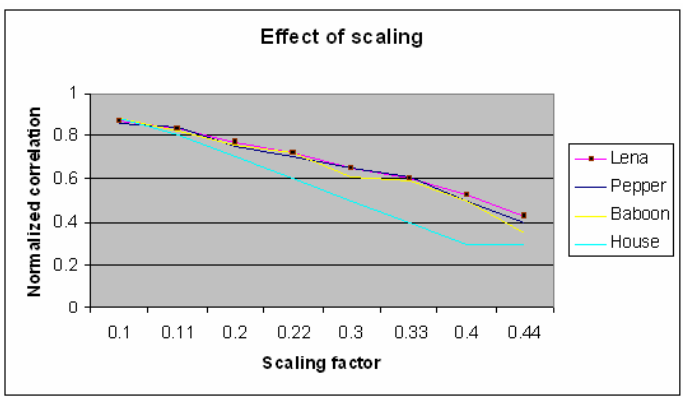

Fig. 13 - Effect of Scaling

\section{E. EFFECT OF BLURRING}

Special type of circular averaging filter is applied on the watermarked color image to analyze the effect of Blurring. The circular averaging (pillbox) filter is applied which filters the watermarked image within the square matrix of side 2(DiskRadius) +1 . The disk radius is varied from 0.6 to 1.4 and the effect of blurring is analyzed on extraction algorithm. Performance of algorithm is decided by calculating $\mathrm{NC}$ between extracted watermark and original watermark. Figure $14 \mathrm{a}$ and $14 \mathrm{~b}$ shows the blurred Lena image and extracted watermark respectively. Figure 15 shows the effect of blurring on watermarked images.
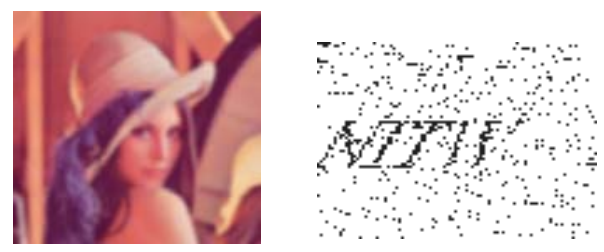

Fig. 14a - Blurred Watermarked Fig. 14b - Extracted image Watermark

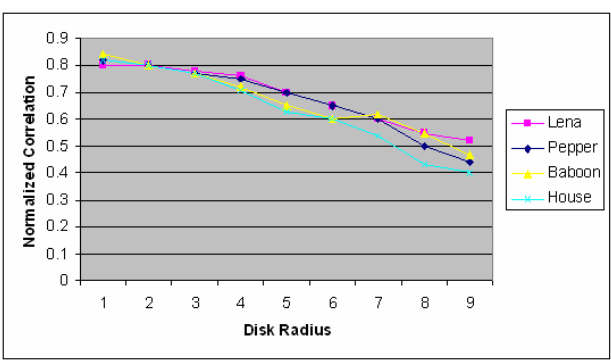

Fig. 15 - Effecy of Blurring on Watermarked images

\section{F. PEAK SIGNAL TO NOISE RATIO}

In experiments, we obtained the third level DWT decomposition using the Haar filter with the value of $\alpha=0.6$. We calculated the amount of noise added into the color (cover) image using Peak Signal to Noise (PSNR) metric.

$$
P S N R=10 \log _{10} \frac{R \times R}{M S E}
$$

Where $R=$ maximum fluctuation in the input

$M S E=\sum_{i=1}^{m} \sum_{j=1}^{n} \sum_{k=1}^{3} \frac{\left(W(i, j)_{k}-W^{\prime}(i, j)_{k}\right)^{2}}{3 m n}$

$=$ height of image, $n=$ width of image, $W(i, j)_{k}$ and $W^{\prime}(i, j)_{k}$ represent $\mathrm{R}, \mathrm{G}, \mathrm{B}$ channels of un-watermarked color image and Watermarked color image respectively. We found that the value of Peak Signal to Noise ratio is $48.52 \mathrm{~dB}$. After extracting the watermark, it is compared with the original watermark by calculating Normalized correlation that is defined below:

$N C=\frac{\sum_{i=1}^{m} \sum_{j=1}^{n} W(i, j) W^{\prime}(i, j)}{\sum_{i=1}^{m} \sum_{j=1}^{n} W(i, j)^{2}}$

Where $W(i, j)=$ original watermark and $W^{\prime}(i, j)$ $=$ Extracted watermark.

\section{F. EFFICIENCY OF ALGORITHM}

To increase the detection speed and efficiency of the algorithm, the location of modified high and middle frequency components are stored into key array. Use of this key array improves the speed of the extraction algorithm. Table 1 shows the time taken by algorithm to extract the watermark with and without key array. Thus by using the key array the speed of extraction is improved.

Table 1. Performance of extraction algorithm with and without key array

\begin{tabular}{|c|c|}
\hline \multicolumn{2}{|c|}{ Time taken by extraction algorithm (in seconds). } \\
\hline Without Key Array & With Key Array \\
\hline 29.12 & 19.76 \\
\hline
\end{tabular}

\section{G. Comparison}

We compare the performance of our algorithm with the other watermarking algorithms based on DWT $[12,15]$. The comparison is decided in Table 2.

As shown in the Table 2, the proposed algorithm has PSNR of $48.32 \mathrm{~dB}$, which is higher than the algorithms used in $[12,15]$. If the watermark is embedded into low frequency components, the low frequency components are more likely to be suppressed by filtration as noise. Hence our algorithm embeds the watermark into high and middle frequency components, which improved the robustness of algorithm against attacks and the 
visual quality of watermarked color images is same as original image. The key array used in extraction algorithm helps to easily identify the modified frequency components and thus improves the speed of extraction.

Table 2. Comparison of proposed algorithm with other DWT algorithm

\begin{tabular}{|c|c|c|c|}
\hline Properties & $\begin{array}{c}\text { Ersin } \\
\text { Elbasi[12] }\end{array}$ & $\begin{array}{c}\text { Ersin Elbasi } \\
{[15]}\end{array}$ & Proposed Algorithm \\
\hline Cover Data & $\begin{array}{l}\text { Gray } \\
\text { Scale }\end{array}$ & $\begin{array}{c}\text { Luminance(y) } \\
\text { channel } \\
\text { of video frame }\end{array}$ & $\begin{array}{c}\text { Luminance }(y) \\
\text { channel } \\
\text { of color image }\end{array}$ \\
\hline $\begin{array}{l}\text { Domain of } \\
\text { embedding }\end{array}$ & $\begin{array}{c}\text { Frequenc } \\
\text { y domain } \\
\text { DWT }\end{array}$ & $\begin{array}{c}\text { Frequency } \\
\text { domain DWT }\end{array}$ & $\begin{array}{c}\text { Frequency domain } \\
\text { DWT }\end{array}$ \\
\hline $\begin{array}{l}\text { Frequency } \\
\text { bands }\end{array}$ & $\begin{array}{c}2 \text { bands } \\
\{\mathrm{LL}, \mathrm{HH}\}\end{array}$ & $\begin{array}{c}\text { All } 4 \\
\text { frequency } \\
\text { components } \\
\{\text { LL,LH,HL,H } \\
\text { H }\}\end{array}$ & $\begin{array}{c}\text { High frequency and } \\
\text { middle components } \\
\{\text { \{L,LH,HH }\}\end{array}$ \\
\hline Watermark & $\begin{array}{l}\text { Pseudo } \\
\text { random } \\
\text { Number } \\
\text { ( PRN) }\end{array}$ & Binary image & $\begin{array}{c}\text { Monochrome image } \\
\text { (logo) }\end{array}$ \\
\hline $\begin{array}{c}\text { PSNR Of } \\
\text { watermarke } \\
\text { d image }\end{array}$ & $40.86 \mathrm{~dB}$ & 42.43dB & $48.32 \mathrm{~dB}$ \\
\hline A & $\begin{array}{l}0.1 \text { for } L L \\
\text { and. } 0.4 \\
\text { for } H H \\
\end{array}$ & ----------- & 0.6 \\
\hline $\begin{array}{l}\text { Input to } \\
\text { extraction } \\
\text { algorithm }\end{array}$ & $\begin{array}{c}\mathrm{T} 1=100, \\
\mathrm{~T} 2=110 \\
\text { for } \mathrm{L} L \\
\text { and } \\
\mathrm{T} 1=30, \\
\mathrm{~T} 2=40 \text { for } \\
\text { HH }\end{array}$ & $\begin{array}{l}\text { Cover video } \\
\text { frame }\end{array}$ & $\begin{array}{c}\text { Key array K } \\
\text { Color (Cover) image }\end{array}$ \\
\hline
\end{tabular}

\section{CONCLUSION}

In this paper we presented a new method of embedding watermark into color image. The scrambled luminance component of image is considered for embedding watermark. On this scrambled luminance component of image DWT is applied to decompose this image into high and middle frequency components. Combinations of high and middle frequency components are considered for watermarking, because the high frequency contains edge information. Further, human eyes are less sensitive to change in the edge. The security in the proposed scheme is achieved using Arnold transform and key array. The strength of algorithm is tested using different types of image processing attacks, like image cropping, image filtering, image compression etc. algorithm is found rigid to different types of attack.

\section{REFERENCES}

[1] A. Piva, F. Bartolini, and M. Barni. Managing copyright in open networks, IEEE Internet Computing, May-June 2002, Vol. 6, Issue 3, pp. $18-26$.

[2] Chun-Shien Lu, Hong-Yuan, and Mark Liao. Multipurpose watermarking for image authentication and protection, IEEE Transactions on Image Processing, Oct 2001, Vol. 10, Issue 10, pp. 1579-1592.

[3] Joo Lee and Sung-Hwan Jung. A survey of watermarking techniques applied to multimedia, Proceedings 2001, IEEE International Symposium on Industrial Electronics (ISIE2001), 2001, Vol. 1, pp. 272277.

[4] I. J. Cox, M. L. Miller, and J. A. Bloom. Digital Watermarking, Morgan Kaufmann Publishers, 2002.

[5] R. G. Schyndel, A. Tirkel, and C. Osborne. A digital watermark, Proceedings of 1994 International Conference on Image Processing (ICIP 1994), Austin, Texas, November 13-16 1994, pp. 86-90.

[6] C. T. Hsu and J. L. Wu. Hidden digital watermarking in images, IEEE Transaction on Image Processing, Jan. 1999, Vol. 8, No. 1, pp. 58-68.

[7] M. Barni, E. Bartolini, V. Cappellini, and A. Piva. A DCT - domain system for robust image watermarking, Signal Processing, May 1998, Vol. 66, No. 3, pp. 357-372.

[8] C. T. Hsu and L. Wu. DCT-based watermarking for video, IEEE Transactions on consumers electronics, Feb. 1998, Vol. 44, No.1, pp. 206-216.

[9] S. Saha, and R. Vcmuri. How do image statistics impact lossy coding performance? Information Technology, 2000, pp. 42-47.

[10] P.-L. Lin. Robust transparent image watermarking system with spatial mechanisms, The Journal of Systems and Software, 2000, pp. 107-116.

[11] J. J. K. O'Ruanaidh, W. J. Dowling, and E. M. Boland. Phase watermarking of digital images, Proceedings of IEEE International conference on Image Processing, Sep. 1996, Vol. 3, pp. 239-242.

[12] Ersin Elbasi and Ahmet M. Eskicioglu. A DWT-based robust semi-blind image watermarking algorithm using two bands, Proc. SPIE, 2006, Vol. 6072.

[13] Chiou-Ting Hsu and Ja-Ling Wu. Hidden Digital watermarks in Images, IEEE Transactions on Image Processing, January 1999, Vol. 8, no. 1. 
[14] Peter Meerwald and Andreas Uhl. A Survey of Wavelet-domain Watermarking Algorithms, Security and Watermarking of Multimedia Contents III, Proceedings SPIE, January 20-26 2001, Vol. 4314, pp. 505-516.

[15] Ersin Elbasi and Ahmet M. Eskicioglu. Robust DWT based MPEG-1 watermarking in four bands, The Second Secure Knowledge Management Workshop (SKM), New York City, NY, September 28-29 2006.

[16] Suhad Hajjara, Moussa Abdallah, Amjad Hudaib. Digital image watermarking using localized biorthogonal wavelets, European Journal of Scientific Research, 2009, Vol. 26, No.4, pp. 594-608. ISSN 1450-216X.

[17] Zhenwei Shang Honge Ren Jian Zhang. A block location scrambling algorithm of digital image based on Arnold transformation, IEEE, 9th International Conference for Young Computer Scientists, 2008, pp. 2942-2947.

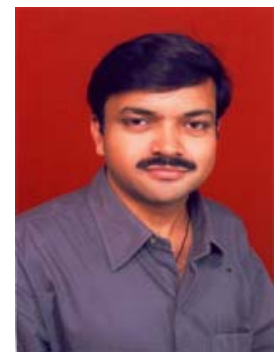

Nagaraj V. Dharwadkar, has obtained B.E. in Computer Science \& Engineering in 2000 and M.Tech. in Computer Science \& Engineering in 2006 $\mathrm{He}$ is Assistant Professor in the department of Computer Science at B.L.D.E.A's Engineering college Bijapur. Presently he is pursuing full time PhD in Computer Science and engineering at National Institute of Technology, Warangal. His research area of interest is Digital Watermarking and image security.

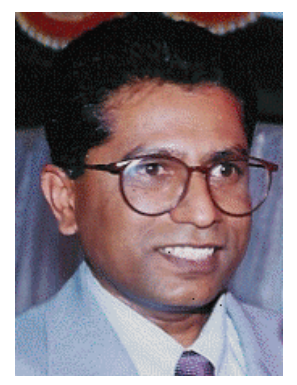

Dr. B. B. Amberker, obtained his Ph.D from the Department of Computer Science and Automation, IISc., Bangalore, India. He is presently working as Professor in the Department of Computer Science and Engineering, National Institute of Technology, Warangal, AP, India. His research area of interest is digital signature, group key communication and Digital Watermarking and image security. 